Philologica Canariensia

Revista de Filología de la Universidad de las Palmas de Gran Canaria

20 (2014), eISSN: 2386-8635

DOI: en trámite

\title{
EXISTE-T-IL UN MACONDO ET DES BUENDÍA \\ QUÉBÉCOIS DANS LE ROMAN HISTORIQUE À LA FIN DU XXE SIÈCLE AU QUÉBEC?
}

\author{
EwELINA BujNOWSKA
}

Université de Silésie (Polonia)

\begin{abstract}
RÉSUMÉ
Dans cet article, la notion de réalisme magique est appliquée à la littérature québécoise. D'abord l'auteure présente le concept afin de bien préciser ses traits caractéristiques. Ensuite, elle analyse le roman historique La tribu de l'écrivain québécois François Barceloen essayant de mettre en lumière son appartenance à cette tendance latino-américaine. Cet examen permet d'enrichir de nouveaux aspectsl'analyse des lettres du Québec. MOTS-CLÉS: réalisme magic, littérature québécoise, roman historique.
\end{abstract}

\section{ABSTRACT}

In the present article, the term "magic realism" is applied to Quebec literature. First the author explains the concept in order to specify its distinctive features. Afterwards she analyses the historical novel La tribu by the Quebec writer, François Barcelo, trying to bring out its affinity to the Latin America's aesthetic style. This examination contributes to enrich the analysis of Quebec literature with new aspects.

KEYWORDS: magic realism, literature in Quebec, historical novel. 


\section{RESUMEN}

En este artículo, aplicamos la noción de realismo mágico a la literatura quebequense. En primer lugar la autora delimita este concepto definiendo sus rasgos característicos, para analizar a continuación la novela histórica $L a$ tribu del escritor quebequense François Barcelo. El estudio trata de esclarecer la pertenencia de esta obra a la citada corriente latinoamericana, lo que permite enriquecer con nuevos enfoques la investigación de la narrativa de Québec.

PALABRAS CLAVE: realismo mágico, literatura quebequesa, novela histórica.

\section{INTRODUCTION}

En tant que concept, le réalisme magique apparaît rarement dans le discours critique concernant la littérature québécoise. L'examen des éléments qui pourraient témoigner de sa présence dans les lettres du Québec nous semble pourtant intéressant afin d'élargir le champ d'analyse de la littérature québécoise et afin de montrer l'étendue du concept. La tribu, roman publié en 1981 par l'auteur québécois François Barcelo, s'approche du modèle réaliste magique et il pourrait être désigné comme appartenant à cette tendance latino-américaine. Avant de passer à l'analyse du texte barcelonien, il est pourtant pertinent d'aborder la réflexion par un bref aperçu sur la notion de réalisme magique elle-même.

\section{LE RÉALISME MAGIQUE : MISE AU POINT}

Comme le remarque Tomasz Pindel, traducteur polonais de la littérature hispanophone, le terme "réalisme magique" (realismo mágico) a fait une carrière éblouissante dans la critique littéraire du $\mathrm{XX}^{\mathrm{e}}$ siècle (Pindel, 211). Malgré cette popularité énorme dont témoigne le succès éditorial foudroyant du texte phare, Cent ans de solitude, l'œuvre la plus traduite et la plus lue en espagnol ${ }^{1}$, les études consacrées au réalisme magique semblent peu nombreuses comme si les universitaires prenaient leurs distances vis-à-vis de cette "formule magique" (Pindel, 213) qui hante la littérature latino-américaine, à ce concept "galvaudé" par la critique littéraire (Le Fustec, en ligne). 
La genèse du terme est européenne. On assigne communément la parenté de l'expression "réalisme magique” à l'Allemand Franz Roh. Ce jeune critique d'art est le premier à utiliser le terme 'Magischer Realismus' dans le titre de son ouvrage Nach-expressionismus, magischer Realismus : Probleme der neuesten europäischen Malerei (Post-expressionnisme, réalisme magique : problèmes de la nouvelle peinture européenne) publié en 1925 (Pindel, 215). Franz Roh emploie cette tournure pour parler d'une nouvelle tendance post-expressionniste, courant pictural d'Europe naissant dans les années 1920. Ce critique d'art allemand désigne ainsi une préférence à rapprocher la magie et le réalisme, un penchant à imaginer "des objets fantastiques" et à associer le quotidien à l'exotisme inopiné (Pindel, 215). Selon Roh, cette peinture a pour but de représenter le merveilleux de la réalité en plaçant les choses et les faits quotidiens dans une perspective et un contexte spécifiques. Presque simultanément en peinture allemande, on commence à utiliser le vocable 'Neue Sachlichkeit' - "nouvelle objectivité" - en référence à l'exposition de Gustav Hartlaub à Mannheim en 1925 (Pindel, 215).

Deux ans après la publication du livre de Franz Roh, le terme est introduit en Amérique hispanique par la Revista de Occidente grâce à José Ortega y Gasset et sa traduction des extraits de Roh d'abord et de l'ouvrage entier ensuite (Pindel, 216). Le Vénézuélien Arturo Uslar Pietri utilise le premier cette appellation dans son essai "El cuento venezolano" dans le recueil Letras y hombres de Venezuela de 1948 pour caractériser l'écriture des jeunes romanciers latino-américains des années 30 et 40 du $\mathrm{XX}^{\mathrm{e}}$ siècle résistant au réalisme (Pindel, 217). Le terme se popularise vite dans les milieux littéraires hispanophones notamment grâce à Alejo Carpentier à qui on attribue la véritable naissance du réalisme magique en lettres latino-américaines (Le Fustec, en ligne). Ce romancier cubain devient le théoricien majeur du réalisme magique dont il pose les bases en 1949 dans le prologue de El reino de este mundo (Le Royaume de ce monde), œuvre de fiction historique, racontant l'histoire d'Haïti. Dès les premiers textes théoriques, on donne au réalisme magique de diverses significations et ses définitions s'appuient sur des fondements très discutés (Pindel, 212).

La publication en 1955 dans la revue new-yorkaise Hispania de l'article "Magic Realism' in Spanish American Fiction" par Angel Flores, universitaire de Queens College, marque le véritable début du débat théorique sur le 
réalisme magique (Pindel, 221). Pour Angel Flores, la nouveauté de la littérature réaliste magique réside dans l'amalgamation du réalisme et du fantastique ("amalgamation of realism and fantasy", cité par Pindel, 222).

Dans les années qui suivent, le débat concerne surtout la dénomination de manière à ce que la question passe "du niveau analytique au niveau terminologique" (Vautier, 1991a, 41). Il existe des textes ou des auteurs que personne n'hésite à classer comme réalistes magiques : Carlos Fuentes, Miguel Angel Asturias, Alejo Carpentier et Gabriel García Márquez. Ils sont probablement les seuls à obtenir l'unanimité quoique cela ne soit pas vrai pour tous leurs ouvrages (Weisgerber, 126). Certains critiques délibèrent s'il faudrait ajouter à ce corpus Juan Rulfo ou encore Jorge Luis Borges ou Julio Cortázar. Maintes divergences parmi les conceptions du réalisme magique découlent évidemment de la diversité des ouvrages considérés comme les textes réalistes magiques.

Bien des théoriciens et universitaires mettent un signe d'égalité entre le réalisme magique et le real maravillloso, réel merveilleux, notion dont Alejo Carpentier est le fondateur. Pourtant comme le remarque Roger Bozzetto :

[c]es deux expressions ne sont pas équivalentes. On pourrait remarquer que le 'real maravilloso' renvoie à l'objet, le monde, en soi "merveilleux" : il institue un rapport original au monde. Par contre le "réalisme magique" fait allusion à un monde de représentation de l'objet. Il renvoie à une esthétique. Mais les œuvres constituent peut-être le lieu et le moyen pour que cette vision du monde et ces tentatives esthétiques se conjoignent. (c'est nous qui soulignons) (Bozzetto, en ligne)

D’après Tomasz Pindel, même si la plupart des caractéristiques du réalisme magique et celles du réel merveilleux ${ }^{2}$ se ressemblent dépendamment des théoriciens, ce qui rend les courants différents l'un de l'autre, c'est la perspective narrative (Pindel, 260).

Alejo Carpentier est lui aussi à l'origine d'une confusion qui nourrit encore à présent le discours littéraire hispanophone quant à la distinction entre le réalisme magique et le réel merveilleux. L’un des obstacles majeurs à définir le réel merveilleux réside dans l'appréhension de la réalité dans le contexte latino-américain. Comme le souligne Alejo Carpentier, le merveilleux du réel, l'étrangeté, reste le patrimoine naturel du continent américain car "tout est merveilleux dans une histoire qu'il est impossible de situer en Europe, et qui 
est tout aussi réelle, toutefois que n'importe quel événement exemplaire consigné, en vue d'une édification pédagogique, dans les manuels scolaires. Mais qu'est ce que l'histoire de l'Amérique si ce n'est pas une chronique du réel-merveilleux?” (cité par Fauchier, 11). Ainsi en Amérique latine, le référent demeure-t-il dissemblable de ceux en Europe ou en Amérique du Nord, car ce qui reste merveilleux pour les Occidentaux relève de "la réalité quotidienne pour beaucoup de peuplades latino-américaines" (Risco, 71).

Pour Carpentier, comme il le souligne dans le prologue du Royaume de ce monde, "la sensation du merveilleux présuppose une foi. Ceux qui ne croient pas aux saints ne peuvent pas être guéris par des miracles de saints..." (Boisclair, Estévez Ruiz, 36). L’un des piliers du réel merveilleux s'avère alors la foi : il faut croire au merveilleux, au surnaturel pour pouvoir céder à sa sollicitation. Par conséquent, dans un texte réaliste magique, l'auteur brosse une collectivité de la classe paysanne d'une époque pré-technologique qui a une foi naïve, chez laquelle la tradition orale demeure toujours vive et laquelle accepte la réalité plus bizarre que la fiction (Vautier, 1998, 206). Le réalisme magique présente alors d'une perspective pré-technologique, voire pré-littéraire (Vautier, 1998, 206), l'univers dans lequel tout est possible, même l'événement le plus bizarre, étrange ou anormal.

Le réalisme magique débouche alors sur le refus de l'opposition européenne classique entre la réalité et l'imaginaire. La notion de réalisme magique, ou plutôt selon les termes de Alejo Carpentier celle de 'real maravilloso americano', vise à s'écarter du fantastique à l'européenne ou à l'anglo-saxonne, à "contrer l'hégémonie culturelle des puissances [...] et leur tendance à définir une 'norme' culturelle" (Le Fustec, en ligne). Afin de ne pas entrer dans un moule littéraire européen et de ne pas chercher, comme les surréalistes et autres "fantastiqueurs", à créer à tout prix le merveilleux, il faut tout simplement observer la réalité américaine car elle comporte en soi cet élément merveilleux. Après son séjour en Europe, Alejo Carpentier confronte ces deux attitudes artistiques :

Après avoir senti le sortilège nullement fallacieux d'Haïti, trouvé des résonances magiques sur les chemins de latérie du Plateau central, entendu les tambours du Pedro et du Rada, je fus tenté de rapprocher la réalité que je venais de vivre de la chasse épuisante au merveilleux qui caractérisa certaines littératures européennes de ces trente dernières années. (c'est nous qui soulignons) (cité par Fauchier, 12). 
Ainsi, d'après le romancier cubain, le fantastique est marqué par l'artifice et par conséquent par le mensonge en dépendant du pacte littéraire entre le lecteur et l'auteur étant chasseur de merveilleux. Par comparaison à 'real maravilloso', le merveilleux auquel les surréalistes ont recours n'est pas “autre chose qu'un subterfuge littéraire qui devient ennuyant à la longue” (Boisclair, Estévez Ruiz, 35). Comme le remarque Alejo Carpentier, présenter le réel merveilleux veut dire décrire une réalité différente de celle connue des Européens, mais pas pour autant moins authentique. Le réel merveilleux semble être, pour cet écrivain, "notre réel merveilleux, tel que nous le trouvons à l'état brut, latent, omniprésent, dans tout ce qui est latino-américain" (cité par Boisclair, Estévez Ruiz, 35). Correspondant aux croyances d'une culture donnée et faisant déjà partie du quotidien, le merveilleux n'est pas création ni pose artistique. L'un des critiques résume cette perception de la réalité et les conséquences qui en découlent comme suit :

\footnotetext{
C'est sans doute pour définir cette réalité hors des normes que les critiques de la génération de Miguel Angel Asturias et Alejo Carpentier ont ajouté au terme réalisme les qualificatifs apparemment contradictoires de "fantastiques" ou de "merveilleux", et plus généralement de "magique". Plus justement aussi, car une imagination souvent effrénée et la recherche (réussie) d’une forme-miroir de cet environnement hallucinant ont fait de ces créateurs des mages de l'écriture. (cité par Fauchier, 13).
}

L'appellation "réalisme magique" semble, tel un oxymore, rapprocher deux termes apparemment contradictoires car "réalisme" et "magique" représentent deux visions du monde opposées, au moins d'un point de vue traditionnel, européen. Alors le but principal d'un écrivain "magico réaliste" n'est pas de copier la réalité, comme le font les réalistes, ni de la transgresser, comme c'est le cas de surréalistes, mais de "capter le mystère palpitant dans les choses" car étant confronté à une réalité autre, l'artiste vise à la dé-faire pour dévoiler du "mystérieux dans les choses, la vie, les actes humains" (Bozzetto, en ligne).

Force est de constater que quasiment tout le problème du réalisme magique se résume à la question-clé pour toute la littérature du continent, à savoir comment parler de la réalité de l'Amérique latine? Depuis l'époque des grandes découvertes, tout au long de la période coloniale jusqu'au XIX siècle, le Nouveau Monde a toujours été regardé à travers les “yeux européens” et la 
littérature a utilisé les modèles provenant de l'Ancien Monde pour en parler. Cette perspective européenne est propre non seulement aux Européens mais aussi aux habitants du Nouveau Monde qui perçoivent leur propre réalité d'une façon stéréotypée en recourant aux moyens caractéristiques des maitres européens (Pindel, 235). Le réalisme magique s'avère alors une issue, un mode nouveau qui rend possible une description autre de la réalité "merveilleuse" américaine.

En s'appuyant sur les travaux des années 1990 visant à théoriser le réalisme magique tels que El realismo mágico, cosmós latinoamericano de Gloria Bautista Gutiérrez de 1991, "Scheherazade’s Children" de Wendy B. Faris, article sorti en 1995 et Historia verdadera del realismo mágico de Seymour Menton publié en 1998, Tomasz Pindel énumère trois caractéristiques principales du texte réaliste magique qui se répètent dans la plupart de ces écrits théoriques récents. Premièrement, la réalité représentée de l'ouvrage réaliste magique ressemble à la réalité du lecteur mais s'y produisent les événements merveilleux, magiques, insolites qui ne semblent pourtant pas violer les lois de la nature et se lient harmonieusement au quotidien. Par contre, la pratique propre à ce genre de textes est de présenter les événements et les faits quotidiens, ordinaires comme s'ils étaient magiques et merveilleux. Deuxièmement, cite Pindel, ce caractère merveilleux compris dans l'univers représenté tire son origine dans une certaine façon de penser, "primitive”, populaire, éloignée du rationalisme, et se réfère au mythe en tant que certaine structure servant à expliquer les mécanismes du monde. Finalement le langage des textes réalistes magiques s'avère simple et clair, vise la précision, évite l'extravagance formelle mais fait souvent preuve d'inventivité (Pindel, 243-244).

Pour couper court les discussions sur les divergences et convergences du réalisme magique et d'autres concepts dont le fantastique ou le merveilleux, Tomasz Pindel constate que: "la littérature du réaliste magique est un phénomène qui se situe entre le merveilleux, le fantastique et le réalisme, elle possède des éléments de tous ces courants mais elle n'appartient entièrement à aucun d'eux" (Pindel, 309 ; notre traduction - E.B.). D'après Antón Risco, il semble indispensable de mentionner que le réalisme magique, ainsi que le réel merveilleux, s'est formé en Amérique latine, contrairement au fantastique, et c'est un concept qui découle de "la volonté pure et simple des critiques de la littérature latino-américaine, de lire le corpus touchant le fantastique ou le 
merveilleux d'une façon particulière, pour ainsi dire autochtone" (Risco, 71) (c'est l'auteur qui souligne).

Bien que les écrivains latino-américains aient longtemps monopolisé l'usage de l'expression 'realismo mágico', de nos jours le réalisme magique gagne du terrain en Occident en tant que mode narratif privilégié et dépasse largement les frontières de l'Amérique latine, sa patrie d'adoption. À présent, les critiques littéraires occidentaux ont tendance à appliquer les notions de réalisme magique et de réel merveilleux aux lettres du monde entier. En pratique littéraire contemporaine, on utilise le plus souvent le réalisme merveilleux afin de commenter les écrits antillais tandis que le terme 'real maravilloso' a le dessus dans les commentaires sur la littérature hispano-américaine et les littératures des pays postcoloniaux non-antillais comme le Canada anglophone ou l'Inde (Vautier, 1991a, 41).

\section{LE RÉALISME MAGIQUE ET LE ROMAN HISTORIQUE}

La plupart des œuvres réalistes magiques sont des fictions historiques ayant leur source dans l'Histoire des patries de leurs auteurs. C'est aussi le cas du texte fondateur d'Alejo Carpentier, le Royaume de ce monde, œuvre très marquée par les faits authentiques tels la révolution haïtienne de la fin du XIX ${ }^{\mathrm{e}}$ siècle, présentant par les yeux du protagoniste Ti Noël, esclave noir, le parcours agité au terme duquel Haïti conquiert son indépendance. Malgré le merveilleux de l'univers représenté, les textes réalistes magiques restent profondément ancrés dans la réalité et mettent en avant une réflexion incessante sur les événements historiques et politiques du Nouveau Monde afin de "désacraliser l'unique version officielle des faits en créant d'autres versions" (Boisclair, Estévez Ruiz, 38). Aussi Gabriel García Márquez dans son Cent ans de solitude s’inspire de l'Histoire pour présenter une fresque de sa Colombie natale et de l'Amérique latine en y incorporant des éléments magiques. Le romancier colombien écrit aussi en 1975 El otoño del patriarca (L'automne du patriarche), l'histoire d'un général "entre cent sept et deux cent trente-deux ans" gouvernant en tyranneau un pays arriéré, quelque part en Amérique du Sud, une sorte de satire de tous les régimes dictatoriaux. D'ailleurs, comme le souligne Antonio Risco, toute l'œuvre romanesque de García Márquez se présente comme "une somme historique onirique de l'Amérique latine dans laquelle le merveilleux - par 
essence inexplicable - peut en même temps signaler l'absurdité du monde [...]" (Risco, 50). Selon lui, après l'auteur de Cent ans de solitude, l'Histoire de ce continent devrait être revisitée car elle reste encore à réécrire.

Selon Alejo Carpentier, au Nouveau Monde, on rencontre au moins deux versions de l'Histoire : celle qui est officielle, le plus souvent inspirée par l'Europe et de nombreuses versions non-officielles. La seconde version prend souvent la forme de réalisme magique où on subvertit la version officielle au moyen de la magie et du mythe (Vautier, 1991b, 49-50). Soucieux de faire comprendre aux lecteurs que l'Histoire reste une des histoires parmi autant d'autres possibles, les auteurs confrontent leurs fictions aux discours officiels (Boisclair, Estévez Ruiz, 38). Ils offrent une vision multiple du passé en interrogeant la fonction de l'Histoire et du récit historique. Comme le remarque Marie Vautier :

[d]ans les œuvres réalistes magiques, on a l'impression que le conteur/narrateur maittrise sa propre version des événements, et que le défi qu'il pose à l'Histoire offre la promesse d'une ré-vision du passé qui n'est pas dominée par une attitude contestataire ou cynique. Le roman réaliste magique ne rejette pas l'Histoire en soi, il s’intéresse à l'Histoire, mais sa façon de redire le passé lance un défi à la version traditionnelle de l'Histoire - version inspirée à l'origine par l'élément colonisateur. (c'est l'auteur qui souligne) (Vautier, 1991a, 42).

C'est là justement que les œuvres réalistes magiques rencontrent les postcolonial studies : enfin, le "colonisé placé hors de l'histoire" (Memmi, 111) peut prendre la parole et offrir sa petite histoire.

D’après Marie Vautier, il existe une différence majeure entre le réalisme magique et 'lo real maravilloso' visible précisément dans leur perception de l'Histoire. Pour elle, le réalisme merveilleux semble toujours affecté d'une vision du monde en partie coloniale dans laquelle on persiste à mettre en doute le discours historique dominant. On s'oppose aux événements du passé ou on adopte une attitude cynique face à l'historiographie, mais on réagit constamment contre l'Histoire, ce qui correspondrait à l'attitude propre au discours décolonisateur. En revanche, le réalisme magique se libère "du discours téléologique et homogène de l'Histoire traditionnelle" et accepte "l'hétérogénéité postmoderne face à l'historiographie et au mythe" (Vautier, 1991a, 42). Ainsi le réalisme magique s'approche-t-il du discours postcolonial 
partageant avec lui la même attitude vis-à-vis de l'Histoire (Vautier, 1991a, 44 et Vautier, 1998, 205).

\section{QU'EST-CE QU'UN TEXTE RÉALISTE MAGIQUE?}

Selon Tomasz Pindel, la plupart des critiques essayant de théoriser le réalisme magique ont fondé leurs réflexions ayant égard à Gabriel García Márquez et son œuvre romanesque (Pindel, 294). Gabo, comme on appelle le romancier en Amérique du Sud, est souvent considéré comme le père du réalisme magique et son influence sur les auteurs réalistes magiques reste indéniable vu la popularité de l'artiste et le succès de son ouvrage le plus lu, Cent ans de solitude. Sa définition du courant embrasse grosso modo celle d'Alejo Carpentier. Aux dires de García Márquez, le réalisme magique est tout simplement le réalisme du continent. D’après lui, en Amérique du Sud, les choses qui semblent inconcevables ailleurs sont possibles. Il suffit de les présenter en faisant appel à un nouveau langage et à de nouvelles techniques de narration (Pindel, 294-295). Cette conviction résulte d'une perception du monde sui generis du nobliste colombien.

Cent ans de solitude, le classique du réalisme magique, ouvrage réaliste magique par excellence, l'un des textes les plus "trompeurs" et les plus "impénétrables" de la littérature contemporaine, est classé parmi d'autres œuvres "infantiles pour adultes" comme Gargantua et Pantagruel, Tristram Shandy, Don Quichotte de la Manche ou Alice de l'autre côté du miroir (Pindel, 294-295). Ce fameux roman est une saga qui présente les péripéties de la famille Buendía fondatrice de Macondo, bourg perdu dans une jungle sud-américaine. Le récit raconte l'histoire de quelques générations des Buendía, qui, selon la prophétie d'un vieux gitan, sont contraints à vivre cent ans de solitude. À travers ce village imaginaire, le narrateur nous plonge dans une fresque spécifique de la Colombie et de tout le continent sud-américain. Aux dires d'un des commentateurs de Gabriel García Márquez, on pourrait considérer Cent ans de solitude comme un microcosme de la société colombienne moderne, comme "une parabole, plus ou moins fidèle à la réalité, du destin de la Colombie, au moins par restriction du peuple latino-américain" (cité par Diop, en ligne) même si dans ce petit village les gens volent sur des tapis volants et les personnes mortes reviennent. Le livre est une synthèse de 
l'Histoire de l'Amérique latine toute entière dès sa découverte par les Européens jusqu'à l'époque actuelle; dès l'époque de l'innocence "primitive", par la période d'influences européennes et de transformations rapides jusqu'à sa décadence (Pindel, 296).

L'un des critiques accentue cet ancrage dans la réalité de l'ouvrage de Gabriel García Márquez et sa corrélation avec la réflexion d'époque comme suit :

On a parlé, à propos de son œuvre, de réalisme magique, ce qui est vrai. Mais je crois que ce qui fait de cette œuvre très engagée et très valable une œuvre finalement très politique, c'est qu'elle décrit la situation non seulement de la Colombie, mais de tous les pays dépendants. García Márquez décrit au niveau de l'univers non conceptuel, cette situation de dépendance : l'isolation de Macondo. (c'est nous qui soulignons) (cité par Diop, en ligne).

Alors les problèmes du continent sont également ceux du petit monde de Maconto et le village connaît les luttes et les conflits représentatifs de la Colombie et de l'Amérique du Sud. Grâce à l'usage des mythes, autochtones aussi bien que bibliques, l'auteur nous présente un panorama social destiné à critiquer des systèmes en usage sur le sol latino-américain, tels le pouvoir politique dictatorial et la domination économique (Diop, en ligne).

Ainsi sur le plan littéraire, on retrouve le réalisme magique lorsque les événements se produisent sur une toile de fond réaliste et vraisemblable. D’après les personnages de l'univers fictif, le monde dans lequel ils fonctionnent est aussi "rationnel" que le nôtre. Même au moment où un fait extraordinaire se manifeste, il n'ébranle pas ce monde et s'insère naturellement dans la trame du récit. Aucun élément ne met en cause le déroulement du fait magique ni son interprétation (Boisclair, Estévez Ruiz, 35).

Dans l'œuvre réaliste magique, le narrateur nous offre un monde où tout est possible et il fait semblant de dépasser les limites assignées à la réalité quotidienne sans pour autant s'efforcer à justifier la plausibilité de ce qu'il présente (Weisgerber, 129). La plupart des faits merveilleux sont donc présentés par le narrateur qui ne suggère aucunement que les merveilles ne sont pas réelles. Au contraire la narration objective et sèche ainsi que la nette distance du narrateur face à l'affabulation font admettre tous les événements comme vrais (Pindel, 298). Cette attitude “imaginante" n'engendre pourtant 
pas une fuite hors de la réalité. Au contraire le dépassement rend possible l'aiguisement de la sensibilité à l'égard de celle-ci (Weisgerber, 129). Certes, Macondo apparaît comme un pays mythique, toutefois l'auteur demeure sensible à l'actualité tout en sollicitant les mythes.

Ce qui est aussi caractéristique de Cent ans de solitude, et d'autres textes réalistes magiques, c'est le fait que le narrateur regarde la réalité à travers les yeux d'un homme formé par la perception du monde "non-occidentale", irrationnelle, "non-civilisée", "non européisée", ceux d'un homme naif du point de vue rationnel, croyant au merveilleux, ne se laissant pas dirigé par le jugement critique logique (Pindel, 210). Il dépeint une communauté où la foi et la tradition orale prédominent et l'intrigue se perd dans des contes magiques, mythiques et allégoriques placés hors du temps et hors de la "réalité" (Vautier, 1991a, 42). Le narrateur recourt à la magie vaudou, à l'imaginaire des peuples primitifs par exemple africains, à la cosmogonie indigène et aux rites païens ainsi qu'à la légende chrétienne coloniale (comme le Christ indien et la Vierge noire). Les croyances, les mythes et les légendes de trois peuples - l'indigène, le Noir et le Blanc - se confondent. Tout cela dans un environnement "exotique", une flore et une faune spécifiques, un habitat abondant (Boisclair, Estévez Ruiz, 35-36).

D’après Tomasz Pindel, nous pouvons parler de deux techniques auxquelles fait appel Gabriel García Márquez dans son fameux écrit, qui font d'un texte un ouvrage réaliste magique : la "mirabilisation du quotidien" et la "quotidiennisation du merveilleux" . La première méthode tend à rendre la quotidienneté merveilleuse et elle consiste à représenter des objets et des événements oridinaires comme s'ils étaient étranges ou bizarres. La deuxième, nommée la "quotidiennisaiton du merveilleux", vise à rendre le merveilleux quotidien et elle se résume à tisser dans la trame des événements fantastiques ou merveilleux. Pourtant ceux-ci sont représentés comme quotidiens et ne bouleversent pas l'ordre du monde. Dans Cent ans de solitude, la première technique est visible lorsque le narrateur présente des objets d'usage quotidien tels le "terrible" dentier de Melquíades, la lunette ou l'amiant, toutes ces "inventions extraordinaires", voudrait-on ajouter d'origine "européenne", qui arrivent au village par l'intermédiaire des Gitans. Quant à la seconde méthode, Gabriel García Márquez l'utilise à profusion : certains personnages vivent trop longtemps, Remedios est élevée au ciel, le Père Nicanor lévite, le jeune 
Auréliano prévoit l'avenir, l'épidémie d'insomnie frappe le village, un des Buendía, Mauricio, est sans cesse suivi de petits papillons jaunes, des habitants de Macondo volent sur des tapis volants, et ainsi de suite.

Le réel se marie facilement au merveilleux car tout est présenté avec une apparente simplicité narrative. Pourtant au moment où le lecteur est prêt à accepter le monde figuré comme merveilleux, de nombreuses données du quotidien le plus banal mettent en parallèle ce monde étranger et étrange avec celui connu du lecteur. Les récits réalistes magiques développent ainsi des éléments historiques et sociaux du quotidien dans une perspective humoristique et déformante.

\section{LA TRIBU - UN ROMAN RÉALISTE MAGIQUE?}

Dans La tribu, comme dans un texte réaliste magique, les événements se déroulent sur une toile de fond réaliste et vraisemblable, pourtant les aventures des héros, membres d'une petite tribu amérindienne, les Clipocs, s'avèrent invraisemblables et peu communes. Avec adresse, le narrateur entrelace les faits majeurs de l'Histoire de la province dans son histoire "fantastique" (Vautier, 1998, 209). Ainsi 'l'univers 'magique' du roman englobe[-t-il] une réflexion soutenue sur les événements historico-politiques du Nouveau Continent" (Vautier, 1991a, 44).

Comme nous l'avons déjà mentionné, le classique du réalisme magique Cent ans de solitude est une saga narrant l'histoire des Buendía du village Macondo. Pourtant au moyen des aventures des membres de cette famille originale, l'auteur retrace l'histoire de la Colombie et de l'Amérique Latine, commençant par sa découverte par les Européens, passant par ses transformations rapides et finissant par sa décadence. De même, la petite tribu barcelonienne synthétise le sort des Premières Nations et celui des Québécois en Amérique du Nord. Dès la dédicace, l'auteur suggère cette interprétation des aventures des Clipocs car il dédicace son œuvre "à toutes les tribus du monde qui tardent à succomber aux tentations de la liberté" et il dénombre les Québécois à côté de neuf nations amérindiennes : ${ }^{4}$

Aux Cris et aux Montagnais, aux Algonquins et aux Hurons, aux Abénakis et aux Micmacs, aux Naskapis et aux Attikameks, aux Mohawks, aux Québécois et à toutes les tribus du monde qui tardent à succomber aux tentations de la liberté. $(T 7)^{5}$ 
Selon Krzysztof Jarosz, cette “dédicace [...] fonctionne, à l’orée de l'œuvre, comme une allégorèse qui permet d'interpréter l'histoire de la petite tribu des Clipocs soit comme telle, soit comme une allégorie de l'histoire des Québécois depuis la découverte du Canada jusqu'à l'époque moderne” (Jarosz, 174-175).

Le récit commence au moment où une expédition "vieux-paysanne", c'est-à-dire française, touche la côte du Nouveau Monde au XVI ${ }^{\mathrm{e}}$ siècle et finit dans la seconde moitié du $\mathrm{XX}^{\mathrm{e}}$ siècle par la signature d'un contrat entre les Clipocs et une certaine Société d'exploitation de la Grande Baie du Nord, accord traitant de questions territoriales. Le narrateur présente les aventures de la tribu qui connait une période de prospérité nomade, qui doit apprendre à cohabiter avec les colons, qui supporte l'oppression "zanglaise", qui est dépossédée de ses droits et de ses terres, qui progresse puis décline, qui connaît des soulèvements patriotiques, des disputes linguistiques de même que des "élections spéciales" où le "non" triomphe. Quoique la "chronique clipocoise" (Jarosz, 172) reste une lecture assez désinvolte de la réalité, il ne s'avère pas difficile de voir bien des analogies entre le monde "clipoc" et la quotidienneté des habitants du Québec et du Canada.

Les personnages barceloniens ressemblent aux contemporains mais ils vivent les aventures que nous ne pouvons pas imaginer. Ainsi, né "sur le contient oriental" (T 69) il y a vingt-cinq mille ans vers la fin d'une grande glaciation, Grand-Nez, avec une poignée de membres de sa tribu à la recherche de la nourriture, fait pendant plusieurs jours une chevauchée effreinée à dos des rennes qui traversent la mer gelée "qu'ils [martèlent] d'un rythme commun, cli-poc-clac-cli-poc-clac" (T 77) pour s'installer sur un territoire nouveau. De même, la tribu fait un "voyage magique". Poussés par l'arrivée de nouveaux voisins, les Clipocs se déplacent de plus en plus vers le nord. Un jour, lors d'une fête organisée pour rendre grâce à la nature au début de l'été, la tribu, établie sur une banquise pour un concours de pêche, se détache du rivage et commence à flotter au gré des courants. Les indigènes entament de cette façon une dérive de trente ans sur "un iceberg très vaste, mesurant près d'une demi-lieue de diamètre” (T 201). Enfin les Clipocs vivent :

[...] à l'abri des agressions qu'elle pouvait commettre ou dont elle pouvait être victime. Dès la première soirée, lorsqu'on perdit de vue le rivage, la tribu se trouva dans un délicieux isolement. Rien ne pouvait l'atteindre, la blesser, la transformer, l'affecter, la gêner, la troubler, la diluer, la provoquer. (T 201) 
Finalement, la tribu retrouve sans le savoir l'endroit précis où elle a déjà vécu "comme si le destin avait vraiment existé et n'avait rien eu de mieux que tourner en rond" (T 218).

Dans cette "saga fantaisiste et désopilante d'une tribu imaginaire" (Michon, 337), "l'histoire d'une bande d'Amérindiens" (Godbout, 129), le romancier part d'une réalité connue du lecteur pour créer un univers fictif qui ne s'éloigne pourtant jamais de la référence première. Sous le voile du merveilleux ou de l'extraordinaire, qui fait penser aux procédés du conte pour enfants, le narrateur juge ses compatriotes, cachés sous des noms curieux ou étranges tels le marquis-général de Trompart et l'amiral Blackburn, qui ressemblent à Montcalm et à Wolfe, acteurs principaux de la bataille des Plaines d'Abraham, et il commente les faits majeurs de l'Histoire québécoise devenus déformés sous la plume barcelonienne comme mentionnée ci-dessus la bataille de la Guerre de Sept ans ou le traité signé par les Clipocs se référant à la première grande entente du $\mathrm{XX}^{\mathrm{e}}$ siècle entre la couronne et les autochtones, la Convention sur la Baie James et le Grand Nord québécois signé par les Cris et les Inuits le 11 novembre 1975. Sous ce voile, le lecteur découvre les traits d'une réalité familière car les problèmes de la province deviennent ceux de la petite société.

Dans une des interviews, François Barcelo affirme d'ailleurs que son deuxième roman est avant tout un roman indépendantiste. Il est à souligner que la production romanesque barcelonnienne a été injustement qualifiée de "fast food littéraire" et la critique n'a pas fait grand cas de sa charge sociale et politique. Entre autres Jacques Godbout souligne à propos de La tribu qu' "[o]n peut [l']aborder avec plaisir" cependant, selon l'écrivain, ce roman "n'a ni structure ni rebondissements" et "[o]n en lit les chapitres comme s'il s'agissait de contes pour enfants qui n'ont de liens entre eux que la voix de l'auteur, ou le temps qui passe" (Godbout, 129). Bien des critiques se sont alors bornés à la forme et au côté divertissant des ouvrages barceloniens. Pourtant comme le remarque François Barcelo au cours d'un entretien:

quoi qu'on puisse penser, mon travail d'écrivain est toujours près de la réalité. [...] je crois que tous mes romans sont des critiques sociales, mais pas déguisées. Il me semble facile de reconnaître dans Agénor... une œuvre pacifiste. La tribu est un roman indépendantiste. Ville-Dieu serait plutôt socialiste. Aaa, Aâh, Ha... est antinucléaire et xénophile. [...] Pourtant je crois être la seule personne à être totalement consciente de ce contenu politique. Aucun critique, à ma connaissance, ne l'a clairement souligné [...]. (Barcelo, 1990, 63-64) 
Avec beaucoup d'humour, le romancier présente la réalité à travers les yeux purs, "primitifs", ceux des Clipocs (Mailhot, 16). Cette conception s'approche de celle des auteurs réalistes magiques car la réalité est ainsi saisie avec les yeux "non européens". L'auteur puise dans l'imaginaire des populations autochtones et les croyances, les mythes et les légendes se confondent.

\section{LES PERSONNAGES RÉALISTES MAGIQUES BARCELONIENS}

Dans La tribu, l'introduction de deux héros, Grand-Nez et Jean-François, baptisé Jafafoua, donne au récit une dimension mythique (Jarosz, 178). Grand-Nez, le premier à être arrivé sur le continent par le détroit de Béring, "se rappelait la vie dans des cavernes humides et froides. Il se rappelait les immenses mammouths qui écrasaient tout sur leur passage. Il se rappelait la faim, la poursuite de plus en plus pénible des troupeaux de rennes de moins en moins nombreux" (T69). De même Jafafoua, le premier "vieux-paysan” à s'être installé sur le territoire autochtone devient par la suite l'ancêtre de tous les Clipocs. Comme le fait pertinemment remarquer Krzysztof Jarosz, les deux personnages symbolisent deux peuples fondateurs du Québec, les autochtones arrivés sur le territoire il y a des milliers d'années et les Français venus s'installer au XVI ${ }^{\mathrm{e}}$ siècle (Jarosz, 178).

À part le recourt aux personnages mythiques et l'immortalité de Grand-Nez, certaines croyances "primitives" s'inscrivent dans la trame du roman. Le comportement de la tribu face à la mort s'approche à celui des peuples non européens. Dans la mentalité occidentale, la mort est séparée de la vie par une ligne de démarcation. Même si les chrétiens ne considèrent pas la mort comme la fin de la vie, mais comme un chemin vers l'au-delà, un passage entre deux vies, l'une terrestre et l'autre éternelle, la crainte de la mort demeure. Elle est regardée comme quelque chose de définitif, d'irréversible. Le monde des "vivants" et celui des "morts" ne se rencontrent pas dans des sociétés dites civilisées et si c'est le cas, la mort évoque l'épouvante et ce contact viole les lois naturelles. La littérature fantastique européenne devient l'expression de cette appréhension et elle abonde en retours des morts auprès des vivants pour semer la terreur (Pindel, 290). Aux yeux de la mentalité "primitive", les limites entre la vie et la mort sont très floues ou s'effacent. Il est donc normal que les morts rendent visite aux vivants. 
Dans le roman, la perception de la mort correspond à celle des "primitifs". Grand-Nez immortel se demande si la mort l'a oublié ou si elle ne lui rend pas visite car le fait d'avoir étranglé le chef des rennes lui a tellement déplu (T 82).

D'ailleurs les Clipocs se comportent également “en sauvages" vis-à-vis la question du temps. Le temps est bouleversé, comprimé et l'on inflige beaucoup de raccourcis à l'Histoire (Vautier, 1991a, 46). Le fragment décrivant le progrès culturel et social que la tribu a fait en est représentatif. Les Clipocs vivent l'évolution en vingt ans tandis que les plus grandes civilisations ont eu besoin de mille ans pour y arriver. Mais comme le souligne le narrateur, ce n'est pas étonnant car cette civilisation a décidé de progresser rapidement et systématiquement et elle a eu pour guide une fille aussi belle qu'intelligente, Mahii (T 138). Ainsi les Clipocs inventent toute une gamme de biens de consommation "indispensables à toute civilisation qui se respecte" (T 140) et ils sont quintuplés en peu de temps. Ce haut niveau de savoir pousse la tribu à massacrer les Niox, tribu voisine, lors d'une bataille féroce. Honteuse de cette cruauté, le chef de la tribu décide de détruire toutes les inventions et de retourner à l'état sauvage primitif.

\section{LA PRÉSENTATION DE L'HISTOIRE}

Les mêmes raccourcis sont imposés à la présentation des faits historiques. D’après Krzysztof Jarosz, "plusieurs fragments de La Tribu constituent de véritables nœuds de polysémie où se superposent plusieurs événements historiques de référence" (Jarosz, 177). À titre d'exemple, citons le chapitre XVIII dans lequel le narrateur entasse en quelques pages événement sur événement : le traité de Paris de 1763, l'Acte de Québec qui serait "une entente tacite entre les vainqueurs et la population des Régions du Haut [le Québec - E.B.] [...] à condition de ne pas tenter de se joindre aux anciennes colonies zanglaises du sud" (T 299), la formation "des groupes de Fils de la Patrie (jeunes Vieux-paysans patriotes)" (T 300) qui semblent évoquer les Fils de la Liberté, association créée avant l'insurrection et "les Enfants de Dieu (jeunes Zanglais fiers de leurs traditions)" qui feraient penser plutôt au Doric Club (T301), le soulèvement de la population "vieux-paysanne" qui correspondrait aux Rébellions de 1837-38, le rapatriement du traité de Lugdune (T 300), 
qui ressemblerait au rapatriement de la constitution en 1982, un référendum pendant lequel 'les 'non' l'emportèrent dans une proportion de trois contre deux" (T 305) (Jarosz, 177). Le temps se voit donc aboli comme dans la plupart des textes réalistes magiques.

Comme dans l'ouvrage de Gabriel García Márquez, le roman finit par la décadence de la tribu. Les Clipocs eux aussi disparaissent successivement : le prophète Notregloire, fils de Mahii et de tous les Clipocs "capables de copulation” (T212), le poète Ksoâr, inventeur de l'écriture et du sonnet, Mahii elle-même, “en ne regrettant rien" (T 340). Ce qui semble absurde, seul l'immortel Grand-Nez survit, bien que condamné à perpétuité pour avoir abattu un hélicoptère et son pilote.

Par l'emploi du merveilleux et par quelques autres procédés caractéristiques du réalisme magique, le roman barcelonien s'inscrit dans la convention du réalisme magique en vue de revisiter le passé du Québéc et du Canada.

\section{LE LANGAGE DANS LA TRIBU}

La tension entre la langue de la population indigène et la langue du colonisateur est soulignée avec acuité dans le roman barcelonien. Pourtant, contrairement à la pratique courante où les textes décrivent la colonisation linguistique du Nouveau Monde, où la langue est imposée et le peuple colonisé est contraint à accepter la langue du colonisateur (Vautier, 1998, 222), la tribu barcelonienne choisit de bon gré de parler français, appelé vieux-paysan, au lieu d'utiliser la langue clipoc, pour aider Jean-François, jeune mousse, "laissé à terre par inadvertance" (T 21) au moment de la découverte du Nouveau Continent. Vu la déficience linguistique de Jean-François, baptisé entre temps Jafafoua, les Clipocs adoptent sa langue car, comme souligne avec ironie le narrateur, "il était fréquent que des tribus entières apprennent le vieux-paysan croyant à tort que c'était la langue des plus forts" (T 24). Ainsi "sans drame ni débats" (Vautier, 1991a, 45), caractéristiques de la société québécoise, ils abandonnent la pratique de leur propre langue :

Étrangement, Jafafoua n'avait appris que quelques mots de la langue des Clipocs, difficile et parsemée de diphtongues imprononçables pour quiconque n'était pas né Clipoc. Par contre, les Clipocs [...] apprenaient avec une facilité étonnante la douce langue du Vieux-Pays, et ils la parlaient sans accent, ou plutôt avec le même 
accent que Jafafoua. Ainsi donc, moins d'un an après l'arrivée de Jafafoua, presque tous les Clipocs parlaient vieux-paysan, alors que Jafafoua ne parlait presque pas clipoc. Non pas que Jafafoua eût été particulièrement inintelligent. Mais il est fort possible, sinon probable, que les Clipocs étaient plus intelligents que lui. (T 56-57)

L'attitude de la tribu s'avère tout à fait différente au moment où les Clipocs croisent sur leur chemin le révérend Nelson Golden, missionnaire "zanglais" qui essaye sans succès de convertir les indigènes au christianisme. Cet évangélisateur manqué, “[qui] n’était pas doué pour les langues et [qui] n’était non plus très intelligent" (T177), ne s'aperçoit même pas que les autochtones parlent la langue "qu'on parlait en Vieux-Pays, tout près de la Zanglemanie dont il était originaire" (T 177). Sans le savoir, Nelson Golden provoque un vif débat linguistique car certains Clipocs désirant lui faciliter la vie suggèrent de changer de langue et d'apprendre celle de l'étranger. Hantés par les discussions incessantes sur la langue, craignant de "se vider de [leur] âme", la plupart des membres de la tribu défendent la pureté du "vieux-paysan" tandis que les autres proposent de laisser le choix à chacun d'apprendre ou non la nouvelle langue :

Plusieurs membres de la tribu furent favorables au projet. Certains soutenaient que changer de langue apporterait un stimulant certain à la vie intellectuelle de la tribu. D'autres prétendaient qu'on pourrait aisément parler deux langues, et que plus on parlerait de langues plus on aurait le cerveau agile. D'autres enfin faisaient valoir que de ces deux langues on finirait par n'en former qu'une seule, et que cette langue nouvelle serait nécessairement plus avantageuse que les deux langues dont elle serait issue, de la même façon que les enfants sont en général plus évolués que leurs parents.

Mais beaucoup de gens s'opposèrent au projet. Certains par déférence envers Jafafoua, que l'on savait incapable d'assimiler une langue nouvelle, puisqu'il en avait été incapable lorsqu'il était arrivé dans la tribu. D'autres craignaient qu'à force de changer de langue la tribu finirait par se vider de son âme, car ils soutenaient que la langue n'est pas que le véhicule de la parole, mais celui de l'être tout entier. D'autres enfin prétendaient que la tribu avait trop changé depuis quelque temps, et que s'il fallait changer de langue chaque fois qu'on rencontrerait un nouvel étranger, on risquait de devenir fou, car chaque nouvel étranger qu'on rencontrait avait sa propre langue. (T 178)

Ce passage parodie les débats linguistiques au Québec des années 1960-70 et souligne, une fois de plus, l'importance de la langue dans le contexte 
canadien. Les arguments des partisans et des adversaires font penser à ceux des tenants et des détracteurs du bilinguisme au Canada. Pourtant, avant que cette "question linguistique" puisse être résolue, le révérend disparaît et meurt quelque temps après dans des circonstances tragi-comiques.

Dans le roman, la langue utilisée par l'auteur est pure et ne recourt pas aux procédés caractéristiques du français québécois, mais elle est inventive. Dans La tribu, les noms de lieux, de personnages et de faits historiques subissent des transformations pour camoufler les vrais référents. Pourtant le voile sous lequel se cache la réalité de référence est à demi transparent et ne sert qu'à rappeler au lecteur qu'il n'est pas question d'un texte réaliste (Jarosz, 175).

Les noms des personnages qui apparaissent dans le roman sont fantaisistes et ludiquement déformés comme c'est le cas de l'amiral Le Corton, voyageur vieux-paysan qui aborde le premier les côtes du Nouveau Continent et d'autres découvreurs tels Poco Poco, découvreur du lointain Orient, le capétan Croom, homme ayant passé un an dans les glaces du Nord, Jeanne Boucher, la première femme ayant traversé le grand désert et Sidi Blachki, découvreur de la source de toutes les mers (T38-39). Les personnages historiques comme le marquis-général de Trompart et l'amiral Blackburn, exigent du lecteur la connaissance des événements majeurs de l'Histoire du pays, mais sont facilement identifiables.

La toponymie québécoise et canadienne se voit également défigurée. Ainsi, la ville de Québec devient sous la plume de François Barcelo, Balbuk où, au début de la colonisation, "il [n']y avait [qu']un fortin de bois rond, un hôpital en pierre, et trois maisons" ( $T$ 95). Ce petit bourg servait aux dames du Vieux-Pays, c'est-à-dire de France, de prétexte pour fonder un hôpital où soigner les indigènes. Le but initial était atteint puisque l'hôpital se remplissait d'autochtones blessés par les militaires qui avaient pour obligation de défendre l'établissement. De plus, l'auteur nous fournit quelques informations permettant de reconnaître le vrai nom de la ville soulignant que Balbuk "était sur la route des fourrures, et dominait un endroit du fleuve où quelques canons suffisaient à empêcher tout navire de remonter plus loin” (T 96). Le Québec apparaît dans le roman comme les Régions du Haut, la mère-patrie s'appelle le Vieux-Pays et la Zanglemanie s'avère la terre d'origine des anglophones, devenus chez François Barcelo, les Zanglais. Dans le texte le Canada ne 
fonctionne pas caché sous des allusions au "gouvernement central". Des territoires européens apparaissent déformés comme, à titre d'exemple, la Xandinavie et les Pays-Creux qui se réfèrent respectivement à la Scandinavie et aux Pays-Bas.

Certes certaines inventions verbales barceloniennes exigent de la part du lecteur la bonne connaissance de la réalité de référence mais "[r]etrouver le vrai nom qui se cache sous son pseudonyme romanesque est dans la plupart des cas un jeu d'enfant et il ne semble pas que Barcelo les ait introduits pour rendre particulièrement ardue la tâche de son lecteur" (Jarosz, 175).

\section{EN GUISE DE CONCLUSION}

L'étiquette "réalisme magique" se rapporte aux ouvrages qui restent ancrés dans la réalité afin de réexaminer les événements historiques et politiques majeurs tout en se référant au merveilleux. Dans ce type de texte, les personnages vivent des aventures insolites, les faits marquants apparaissent déformés et le temps semble aboli. L'aspect merveilleux semble déterminer l'œuvre de François Barcelo de 1981.

D’après Petr Kyloušek, "la présence du merveilleux dans le roman québécois n'est pas un fait nouveau. Dès les années 1960, notamment, il marque l'œuvre romanesque de Jacques Ferron, mais aussi d'autres romanciers tels Michel Tremblay ou Yves Beauchemin.” (Kyloušek, 100). Selon Kyloušek, trois facteurs structurels rendent possible la rencontre du romanesque et du merveilleux dans le contexte québécois. Premièrement, la forte position dans la culture québécoise de l'oralité dérivant du folklore. Deuxièmement, un fondement ontologique spécifique du conte merveilleux né au Canada français et au Québec, par rapport au conte merveilleux d'origine européenne, qui se situe à mi-chemin entre le récit merveilleux d’inspiration folklorique et le conte fantastique. Troisièmement, à partir des années 1960 l'intérêt que témoignent certains écrivains québécois à l'oralité, à l'imaginaire et aux traditions populaires en y cherchant un nouveau moyen d'expression semble grandir (Kyloušek, 100).

Dans le roman québécois le recours au merveilleux rencontre la tendance dominant les lettres d'Amérique latine, à savoir le réalisme magique. Traditionnellement utilisé pour parler de la littérature sud-américaine, le 
concept s'étend de plus en plus à d'autres littératures du monde entier et permet d'élargir leur analyse par de nouveaux aspects.

\section{NOTAS}

1 L'œuvre de García Márquez a été traduite en 35 langues et vendue, depuis sa publication en 1967, à 30 millions d’exemplaires à travers le monde. ("García Márquez fête ses 'Cent quarante ans de solitude' », 20).

2 Avant tout, les deux, réalisme magique et réel merveilleux, prennent racine dans le surréalisme et utilisent la même technique d'esthétiser pour présenter la réalité. ("Realizm magiczny i lo real maravilloso posiadają niewątpliwie szereg cech wspólnych - surrealistyczne korzenie i technikę estetyzacji opisywanej rzeczywistości - niemniej wykazują zasadnicze rozbieżności pod względem narratorskiej perspektywy” (Pindel, 260).

3 Afin de bien rendre compte de ces deux techniques, Tomasz Pindel forge les néologismes, 'ucudownianie codzienności' et 'ucodziennianie cudowności', que nous proposons de traduire par la "mirabilisation du quotidien" et la "quotidiennisation du merveilleux”. (Pindel, 301).

4 Il est à noter que l'auteur omet dans cette énumération les Malécites. Les membres de cette communauté autochtone vivent sur le territoire québécois. Ceci pourrait s'expliquer par le fait que l'Assemblée nationale a reconnu officiellement les Malécites comme onzième nation autochtone au Québec, y compris les Inuit, en 1989, soit huit ans après la publication du livre de François Barcelo. (Secrétariat aux affaires autochtones : en ligne).

5 Toutes les citations de ce roman se référeront à l'édition de 1998 et seront signalées par le sigle T, suivi du numéro de la page.

6 Le narrateur dresse une longue liste d'inventions clipocs : "la machine à vapeur, la pile de Volta, les toilettes à eau, le chauffe-eau au gaz, le ping-pong, le transistor, le métier à tisser mécanique, les pâtes alimentaires, la montgolfière, l'imprimerie, le canon de 75 milimètres, la carabine à répétition, le yoyo, les préservatifs, la pizza, l'allumette, la brouette, la poubelle à pédale, la photographie, la corde à linge, la planche à laver, le chauffage central, le fer à friser, la bière d'épinette, les allocations familiales, le téléphone-jouet, le moulinet à tambour, le houla-houp, le cure-dents, la baïonette, la mayonnaise, les plombages dentaires, la herse mécanique, les engrais azotés, la machine à écrire, l'assurance-chômage, les échasses, la loupe, l'insémination artificielle, le coït interrompu, les jeans collants, la crème glacée, le réfrigérateur électrique, le presse-ail, la peinture à l'huile et mille et une autres choses" (T 140). 


\section{REFERENCIAS BIBLIOGRÁFICAS}

BARCELO, F. 1990. "Je suis un écrivain, un point c'est tout", propos recueillis par Claude Grégoire, Québec français, nº 78 (été) : 63-64.

BARCELo, F. 1998. La tribu. Montréal : Bibliothèque Québécoise.

Boisclair, M. et Estévez Ruiz, M. 2003. "Le roman et le conte hispano-américains", Québec français, $\mathrm{n}^{\circ} 129$ (printemps) : 32-39.

Diop, E. H. A. 2002. "Le réalisme social et magique chez Gabriel García Márquez et chez Sony Labou Tansi”, Ethiopiques, 68, 1er semestre,

(http://ethiopiques.refer.sn/spip.php?article291) [consulté le 14 août 2010].

FAuchier, J. 2002. Le "réel merveilleux" chez. Alejo Carpentier, René Depestre et Gabriel García Márquez, Thèse de doctorat, Université de la Réunion.

“García Márquez fête ses 'Cent quarante ans de solitude”, Haïti Progrès, vol. 25, numéro 01 du 14 au 20 mars 2007 : 20. (http://www.haitiprogres.com/pdf/H01P20.pdf) [consulté le 4 août 2010].

GodBout, J. 1982. “Où est passé le Barcelo d'Agénor?”, L'Actualité, vol. 7, nº 5 (mai) : 129.

Jarosz, K. 2005. "La Tribu de François Barcelo. Entre un roman historique et une histoire romanesque” en Paluszkiewicz-Misiaczek, Magdalena, Reczyńska Anna, Śpiewak, Anna (coords.) Lien et Mémoire an Canada : Perspectives globales, Kraków : Polska Akademia Umiejętności : 169-180.

KYLOUŠEK, P. 2007. "Le merveilleux et la fiction dans le roman historique La Terre promise, Remember! de Noël Audet”, Études romanes de Brno, Brno : Masarykova univerzita v Brně, 37, $1: 99-111$.

Le Fustec, C. 2010. "Le réalisme magique : vers un nouvel imaginaire de l'autre?”, Amerika [En ligne], 2 |2010, mis en ligne le 25 juillet 2010 (http://amerika.revues.org/1164) [consulté le 26 juillet 2010].

Мапнот, M. 1982. “François Barcelo : La Tribu”, Le Droit, 30 janvier, vol. 69, nº 255 : 16.

Memmi, A. 2002. Portrait du colonisé précédé de Portrait du colonisateur et d'une préface de Jean-Paul Sartre, Paris : Gallimard.

Michon, J. 1982. "Romans" : Lettres in Canada 1981, University of Toronto Quarterly, vol. $51, \mathrm{n}^{\circ} 4$ (Summer) : 334-343.

PINDEL, T. 2004. Zjany, szaleństwo i śmierć. Fantastyka $i$ realizm magiczny w literaturze bispanoamerykańskiej. Kraków : Towarzystwo Autorów i Wydawców Prac Naukowych "Universitas", Studia latynoamerykańskie Uniwersytetu Jagiellońskiego.

Risco, A. 1983. "Le fantastique en Amérique latine : Gabriel García Márquez", Québec francais, $\mathrm{n}^{\circ} 50: 48-50$. Secrétariat aux affaires autochtones,

(http://www.saa.gouv.qc.ca/relations_autochtones/profils_nations/malecites.htm) [consulté le 20 février 2011].

VAUTIER, M. 1991a. "La révision postcoloniale de l'histoire et l'exemple réaliste magique de François Barcelo." Studies in Canadian Literarure/Études en littérature canadienne, vol. $16, n^{\circ} 2: 39-53$. 
VAUTIER, M. 1991b. "Le mythe postmoderne dans quelques romans historiographiques québécois”, Québec Studies, no12 : 49-57.

Vautier, M. 1998. New World Myth. Postmodernism \& Postcolonialism in Canadian Fiction. Montreal \& Kingston : London Buffalo, McGill- Queen's University-Press.

Weisgerber, J. 1987. Le réalisme magique. Roman, peinture, cinéma. Centre d'étude des avant-gardes littéraires de l'Université libre de Bruxelles : Éditions l'Age d'homme. 